Invited review

\title{
Genetic approaches in Drosophila for the study neurodevelopmental disorders
}

\author{
Zeynep Okray a,b,c, Bassem A. Hassan ${ }^{\mathrm{a}, \mathrm{b}, \mathrm{c}, *}$ \\ ${ }^{a}$ Laboratory of Neurogenetics, VIB Center for the Biology of Disease, VIB, Herestraat 49, Leuven, Belgium \\ ${ }^{\mathrm{b}}$ Center for Human Genetics, University of Leuven School of Medicine, Leuven, Belgium \\ ${ }^{\mathrm{c}}$ Program in Molecular and Developmental Genetics, Doctoral School of Biomedical Sciences, University of Leuven, Leuven, Belgium
}

\section{A R T I C L E I N F O}

Article history:

Received 13 April 2012

Received in revised form

31 August 2012

Accepted 7 September 2012

\section{Keywords:}

Intellectual disability

Neurodevelopmental disorders

Drosophila genetics

\begin{abstract}
A B S T R A C T
The fruit fly Drosophila melanogaster is one of the premier genetic model organisms used in biomedical research today owing to the extraordinary power of its genetic tool-kit. Made famous by numerous seminal discoveries of basic developmental mechanisms and behavioral genetics, the power of fruit fly genetics is becoming increasingly applied to questions directly relevant to human health. In this review we discuss how Drosophila research is applied to address major questions in neurodevelopmental disorders.
\end{abstract}

This article is part of the Special Issue entitled 'Neurodevelopmental Disorders'.

(c) 2012 Elsevier Ltd. All rights reserved.

\section{Introduction}

The nervous system starts out as a flat sheet of cells within the embryo, which then folds, twists and grows into a mature organ with hundreds of billions of cells organized into functional units to receive, process and respond to information. During this process, complex genetic and molecular interactions ensure that all cells follow a specified developmental program that leads to their appropriate role in the system. Defects in the development and maintenance of a healthy nervous system can compromise cognitive abilities in humans, with most of these defects leading to diagnoses of neurodevelopmental disorders (NDD) characterized by intellectual disability (ID). How genetic mutations affect the development of the brain by disrupting molecular and cellular networks, ultimately contributing to NDD phenotypes, are large outstanding questions requiring a fundamental understanding of how the brain works. To that end, several animal models are used to study neural development in detail and help provide answers to these questions.

When using animal models, there is usually a trade-off between experimental tractability and anatomical and functional relevance to human disease. The fruit fly, Drosophila melanogaster, offers an effective solution to this tradeoff. It serves as a powerful tool for studying brain development and related disorders, as the principles of nervous system development and function are conserved across

\footnotetext{
* Corresponding author. Laboratory of Neurogenetics, VIB Center for the Biology of Disease, VIB, Herestraat 49, Leuven, Belgium.

E-mail address: Bassem.Hassan@cme.vib-kuleuven.be (B.A. Hassan).
}

lineages. Moreover, its experimental tractability allows rigorous mechanistic analysis and discovery of brain function. This tractability extends first from the simplification of the biology of the fly: with less genetic, cellular and behavioral complexity, it is easier to establish cause-effect relationships in the fly than in higher organisms such as the mouse. Further, the genetic tools available to fly researchers are unparalleled in any organism short of the baker's yeast.

In this review, we discuss how conserved processes between humans and Drosophila make the fly a powerful model to study human NDDs. We go on to describe genetic tools used to investigate fly brain structure and function and give demonstrative examples of discoveries in the fly that provide insight into the mechanisms underlying human cognitive disorders. We seek to highlight the power of the fly as a tool for understanding the etiology of NDDs, however it is not within the scope of this manuscript to extensively catalog discoveries made in the fly, nor is it to outline the more technical details of experimental approaches used in Drosophila. For a comprehensive review of fly tools used in the study of the brain, see Venken et al. (2011) and for a more comprehensive reviews of NDD-related genes studied in Drosophila, refer to reviews by Restifo (2005), Bolduc and Tully (2009), Gatto and Broadie (2011).

\section{Drosophila and mammals share the basic building blocks necessary for brain function and behavior}

Many principles of the nervous system seem to have remained unchanged across evolutionary lineages. This has led to the 
hypothesis that all animals with nervous systems originate from a single ancestor that possessed a sophisticated and highly robust nervous system, developmentally stable enough to withstand evolutionary changes without altering basic structure and function (Ghysen, 2003). The use of animal models, and D. melanogaster in particular, to understand the genetic and molecular mechanisms of NDDs hinges on this fact.

For example, flies and humans share, to a considerable extent, the genetic inventory necessary for building a brain (Thor, 1995). In the context of human disease and disorders, up to two thirds of human disease genes reported in the Online Mendelian Inheritance in Man (OMIM) database have a homolog in the fruit fly (Reiter et al., 2001) and the conservation of ID genes is particularly high: up to $87 \%$ have D. melanogaster homologs (Inlow and Restifo, 2004). In fact, several human ID genes have been named after their Drosophila homologs since mutant phenotypes for these genes were first identified in the fly prior their discovery as ID genes in humans (Inlow and Restifo, 2004). The identification of MR genes and their cataloging in OMIM is reviewed in depth by Inlow and Restifo (2004), with a particular focus on Drosophila homologs. A valuable online tool is Homophila, a cross-genomic database that lists fruit fly homologs of all known human disease genes registered in OMIM (Chien et al., 2002) (Table 1).
Within the context of genetic homology, we can already begin to mention the tractability of the fly as a model system. The genomes of mammalian models are more complex than Drosophila in part because they bear larger gene families. Members of a gene family can be functionally redundant, or a common function can be partitioned among paralogs. As an evolutionary mechanism, this buffers against genetic mutations and allows innovation of new function from extant genes (Ohno, 1970), but in practice this makes mammals more challenging models for understanding genome function. For example, many genes linked to NDDs have several paralogs in humans, while in the fly, are found as only a single homolog. Specifically, $f x r-1$ and $f x r-2$ are two paralogs of the Fragile-X mental retardation ( $f m r-1)$ gene in humans and mice, whereas Drosophila has a single fmr-like gene (Wan et al., 2000). Reducing genetic complexity introduced by post-transcriptional processing further aids in studying the genetic basis of NDDs as it may unmask mutations causing subtle phenotypes. For example the mRNA MAPK-activated ribosomal S6 Kinase (rsk) - mutations for which are associated with Coffin-Lowry syndrome - has four isoforms in humans, while Drosophila has only one (Romeo et al., 2012).

The genes central to fly neural development are functionally conserved in mammals and take part in similar neurobiological

Table 1

Major NDDs modeled in the fly.

\begin{tabular}{|c|c|c|c|c|}
\hline Neurodevelopmental disorder & $\begin{array}{l}\text { Human gene } \\
\text { affected }\end{array}$ & $\begin{array}{l}\text { Drosophila } \\
\text { homolog }\end{array}$ & Gene function in Drosophila & References \\
\hline Fragile X Syndrome (FXS) & Fmr1 & dfmr1 & $\begin{array}{l}\text { Molecular function: RNA-binding protein known to } \\
\text { regulate a large set of mRNA targets. } \\
\text { Biological process: fine tunes synaptic transmission } \\
\text { and neuronal excitability. } \\
\text { Involved in axonal/dendritic growth and elaboration, } \\
\text { axon guidance, neuronal pruning, synaptogenesis. } \\
\text { Control of cell cycle during embryogenesis. } \\
\text { Differentiation and proliferation of neuroblasts in } \\
\text { the developing brain. } \\
\text { Differentiation and proliferation in the germline. } \\
\text { Behavioral phenotypes in mutant: } \\
\text { Include defects in circadian rhythm, memory and } \\
\text { learning, courtship. }\end{array}$ & $\begin{array}{l}\text { Wan et al. (2000), Zhang et al. (2001), } \\
\text { Morales et al. (2002), Schenck et al. (2003), } \\
\text { Pan et al. (2004), Reeve et al. (2005), } \\
\text { Michel et al. (2004), Lee et al. (2003), } \\
\text { Xu et al. (2008), Papoulas et al. (2010), } \\
\text { Costa et al. (2005), Epstein et al. (2009), } \\
\text { Dockendorff et al. (2002), Inoue et al. (2002), } \\
\text { Bolduc et al. (2008), Banerjee et al. (2010), } \\
\text { Coffee et al. (2011) }\end{array}$ \\
\hline Angelman syndrome (AS) & Ube3a & dube3a & $\begin{array}{l}\text { Molecular function: E3 ubiquitin-protein ligase. } \\
\text { Biological process: dube3a activity linked to the } \\
\text { dopaminergic pathway via interaction with Punch, } \\
\text { a regulator of monoamine synthesis. } \\
\text { Loss of function of dUbe3a associated with defects } \\
\text { in dendritic architecture. } \\
\text { Behavioral phenotypes in mutant: Include defects } \\
\text { in memory, locomotion/climbing and circadian rhythm. }\end{array}$ & $\begin{array}{l}\text { Wu et al. (2008), } \\
\text { Lu et al. (2009), } \\
\text { Ferdousy et al. (2011) }\end{array}$ \\
\hline Neurofibromatosis type 1 & $\begin{array}{l}\text { Neurofibromin } 1 \\
\text { (NF1) }\end{array}$ & dNf1 & $\begin{array}{l}\text { Molecular function: Ras GTPase activating protein. } \\
\text { Biological process: affects Ras-dependent and CAMP } \\
\text { dependent pathways. } \\
\text { Mutants show loss of cell cycle inhibition. } \\
\text { Behavioral phenotypes in mutant: } \\
\text { Include defects in learning and memory, escape fly } \\
\text { response and circadian rhythm. }\end{array}$ & $\begin{array}{l}\text { The et al. (1997), } \\
\text { Guo et al. (1997), } \\
\text { Guo et al. (2000), } \\
\text { Buchanan and Davis, (2010), } \\
\text { Williams et al. (2001) }\end{array}$ \\
\hline $\begin{array}{l}\text { Periventricular nodular } \\
\text { heterotropia }\end{array}$ & Filamin A & cheerio & $\begin{array}{l}\text { Molecular function: actin-binding protein. } \\
\text { Biological process: regulates cytoskeleton remodeling. } \\
\text { Involved in axon guidance of motor neurons. } \\
\text { Loss of function affects ovarian structure and oogenesis. } \\
\text { Inhibits lamellocyte differentiation. } \\
\text { Behavioral phenotypes in mutant: } \\
\text { Include defects in memory. }\end{array}$ & $\begin{array}{l}\text { Zheng et al. (2011), } \\
\text { Robinson et al. (1997), } \\
\text { Sokol and Cooley (1999), } \\
\text { Sokol and Cooley (2003), } \\
\text { Rus et al. (2006), } \\
\text { Dubnau et al. (2003) }\end{array}$ \\
\hline Coffin-Lowry syndrome (CLS) & Rsk II & dRsk & $\begin{array}{l}\text { Molecular function: Ribosomal protein S6 kinase II. } \\
\text { Biological process: involved in regulation of the } \\
\text { Erk/MAPK pathway. } \\
\text { Negative regulator of cellular differentiation. } \\
\text { Loss of function affects bouton formation in the } \\
\text { neuromuscular junction. } \\
\text { Behavioral phenotypes: } \\
\text { Include defects in learning and memory, and fine } \\
\text { tuning of circadian rhythm. }\end{array}$ & $\begin{array}{l}\text { Kim et al. (2006), } \\
\text { Fischer et al. (2009), } \\
\text { Putz et al. (2004), } \\
\text { Akten et al. (2009) }\end{array}$ \\
\hline
\end{tabular}


processes such as neuronal differentiation and cellular function (Bellen et al., 2010; Reichert, 2002; Reichert and Simeone, 1999; Rubin et al., 2000). Strong demonstrations of this have come from studies of genetic complementation, in which a gene from Drosophila can functionally replace its mammalian homolog or vice-versa (Bonini, 2000). For example, the mouse and human gene math1, critical for normal neuronal development, has a counterpart, atonal, first discovered in flies that can completely functionally complement CNS and PNS defects in math1-deficient mice (Wang et al., 2002). In turn, atonal mutant phenotypes can be reversed with the expression of math1 in the fly (Wang et al., 2002). Another example is the otx gene family, disruptions in which lead to brain abnormalities including reduced brain size and neuronal defects that ultimately cause epileptic seizures in mice (Acampora et al., 1996; Sancini et al., 2001). Mouse otx1 ortholog can alleviate the corollary phenotype in flies - loss of anterior head structures - and likewise many of the mouse phenotypes are complemented by the fly homolog otd (Nagaod et al., 1998; Acampora et al., 1998).

Along with a more complex genome, the interconnections between neurons of the human brain are orders of magnitude more complex than that of the fly brain. Again, despite the vast organizational differences in brain anatomy, the fly offers a powerful simplification of human NDDs. This is because the cellular components of mammalian and fly nervous systems are structurally and developmentally comparable (Araujo and Tear, 2003; Sanchez-Soriano et al., 2005; Hirth et al., 2003). For example, the fly brain has a level of diversity and complexity of cell-types similar to that of the human brain, however it is a more tractable system because there are fewer total cells to work with. In fact, the fly's brain is small enough that one can microscopically peer at every neuron at once in a single sample.

Furthermore, despite the more complex wiring patterns in mammalian neurons, the mechanisms cells use to connect with one another are evolutionarily conserved. Like mammalian neurons, fly neurons have axons and dendrites that follow evolutionarily conserved molecular guidance cues - such as Netrins, Slits, Semaphorins, and Ephrins - to extend to and connect with their synaptic targets (Dickson, 2002). Once connected, fly and human neurons use the same mechanisms for inter-cellular communication, such as action potentials and neurotransmitter signaling. Evolutionarily conserved $\mathrm{Na}+, \mathrm{K}+$, and $\mathrm{Ca}++$ ion channels maintain cell voltage and regulate firing of action potentials (Littleton and Ganetzky, 2000). Shared vesicular trafficking mechanisms allow the release of universal neurotransmitters such as GABA, glutamate and acetylcholine to mediate synaptic signaling (Littleton, 2000; Nichols, 2006). Interestingly, the fly brain can respond to human psychoactive drugs such as ethanol and cocaine, further suggesting conservation of molecular pathways underlying brain function at the network level (Heberlein, 2000; Heberlein et al., 2009). In fact, the Drosophila model serves as a valuable platform for neuropharmacological research and has been successfully used to identify therapeutic approaches to several brain disorders such as Huntington's and Fragile X Syndrome (Nichols, 2006; Steffan et al., 2001; McBride et al., 2005; Choi et al., 2010; Manev et al., 2003). These findings have provided impetus for similar studies in mouse models and clinical trials with Fragile X patients.

NDDs are essentially characterized by defects in cognition and behavior, and a functioning human brain presumably generates more sophisticated cognitive output than that of a fly. These sophisticated cognitive functions allow us to have emotions, nurture our young and plan for the future. Nevertheless, the fly is capable of its own suite of complicated behaviors (Greenspan and van Swinderen, 2004), and the most basic behaviors of humans and flies are again shared. The plasticity of their nervous systems allows flies to habituate to sensory stimuli, and learn and form memories of associations through various training modules (Pitman et al., 2009). Flies have diurnally rhythmic activity patterns, and require sleep for proper brain function (Hardin, 2005; Cirelli and Bushey, 2008). They also have social behaviors that can be systematically assayed, such as sophisticated courtship rituals and aggressive interactions fueled by hunger or the desire to mate (Dankert et al., 2009). These albeit simple examples are nonetheless the most conserved and critical functions required for survival, and have been extensively studied in flies.

Perhaps more importantly, many genetic and molecular underpinnings of behavior are shared between humans and flies (Greenspan and Dierick, 2004). The genes driving human NDDs like Fragile X Syndrome, Kleefstra Syndrome, Neurofibromatosis type 1, Angelman Syndrome, MRT1 and Coffin-Lowry Syndrome have homologs in the fly that lead to learning and memory defects akin to those in humans (McBride et al., 2005; Kramer et al., ; Guo et al., 2000; Wu et al., 2008; Didelot et al., 2006; Putz et al., 2004). This makes the fly relevant to the study how functional disruptions in these genes can lead to ID-like deficits. Furthermore, the fly can serve as a platform for the de novo discovery of genes likely to underpin human cognitive disorders (Inlow and Restifo, 2004; Morley and Montgomery, 2001), and thereby contribute significantly to our understanding of neurobiological processes relevant in human behavioral disorders. Indeed, the study of behavioral neurogenetics in flies - pioneered by Seymour Benzer in 1960s led to the isolation of many key genes that affect neuronal functions involved in circadian rhythm, learning and memory and has laid the foundations for our understanding of many human neurological processes (Bellen et al., 2010; Vosshall, 2007). For example, identified in the behavioral screens of Benzer, dunce (Dudai et al., 1976) and rutabaga mutants led to the discovery of the role that cAMP synthesis plays in learning and memory, a pathway implicated in several NDDs in humans (Kelley et al., 2007; Al-Tawashi et al.; Mironov et al.; Bourtchouladze et al., 2003).

\section{Experimental approaches in Drosophila}

Nervous system development and function can be studied on many different scales, starting from the biophysical and molecular interactions in single cells to the behavior and social interactions of the organism. Due to its exceptional experimental tractability, the fruit fly provides one of the most powerful models to explore these scales. A continuously expanding genetic toolbox, the use of wellestablished anatomical probes, biochemical assays, live imaging techniques, physiological recordings, and a battery of behavioral tests allow the study of fly brain structure and function in its entirety (Venken et al., 2011). In the following sections, we outline the use of genetic experimental approaches.

\section{Identification and analysis of new genes}

Flies are effective tools for the discovery of novel genes involved in nervous system development and function. Their genetic background and environmental conditions can be tightly controlled, and because they are highly fertile and have short generation times, they can be analyzed in large enough numbers to detect rare phenotypes. This is particularly important in the study of NDDs, since these disorders are dramatically influenced by synergistic genetic and environmental interactions, and thus typically occur with variable penetrance and expressivity. Moreover, the largely unbiased nature of genetic screens in Drosophila allows for insights into mechanisms that may be relevant to disease pathology without prior knowledge or potentially erroneous assumptions. 
Two approaches are commonly used to identify gene function. In forward genetic screens, randomly mutagenized lines of flies are screened for disruptions in a particular phenotype (such as defects in learning and memory). In contrast, reverse genetic approaches focus on identifying the phenotypic consequences of altering specific genes.

For forward genetic screens, libraries of flies are first created by random mutagenesis using chemicals, irradiation or transposon-mediated genetic disruptions (St Johnston, 2002). After generation of the library, it is the researchers' task to screen individual mutants for defects in a function of interest. Using this approach, hundreds of genes involved with the development of particular anatomical features or behavioral deficits have been discovered. The challenge, however, lies in identifying the nature and genomic position of the randomly disrupted gene. This is particularly true for the standard chemical mutagen used in the fly, called ethylmethane sulfonate (EMS). EMS treatment is easy to administer and produces frequent point mutations at random that can saturate the entire genome. Because point mutations can lead to partial or complete loss of function, this approach can unveil the role played by genes essential for the survival of the organism. Furthermore, chemical mutagenesis can offer insight into critical residues required for gene function. Unfortunately, it has traditionally been difficult to map and unravel the specific mutations underlying the identified phenotype. However, this mutagen remains popular today due to the recent development of approaches that exploit the power of whole-genome sequencing.

An alternative way to screen for novel genes is via transposonmediated mutagenesis. To generate the mutant library, flies are first induced to have genetically engineered transposable elements insert throughout the genome, disrupting one or a few genes. Then traditional screening approaches are used to identify affected phenotypes. One advantage to this approach is that transposon insertions typically result in partial loss of function, which allows the study of genes that are required for survival. Moreover, transposon mutagenesis is particularly effective in the search for new genes because the genomic location of the insertions can be easily recovered using standard laboratory techniques. There are already extensive collections of transposon insertion lines available for screening and functional analysis.

While forward screens are used in the discovery of novel genes involved with a given phenotype, reverse genetic screens help to explore the function of a particular gene, such as a homolog of a known human disease gene (St Johnston, 2002). Using reverse genetic strategies, a gene is targeted for disruption, and subsequently the researcher goes on to characterize the phenotypic consequences. In the fly, genes can be disrupted by deletions, RNAi or by site-specific knock-out and knock-ins. Transposon libraries are also used in reverse genetic approaches: genomic lesions can be generated either by inducing the imprecise excision of the transposon or by the splicing of sequences flanked by two different transposons. Currently, deletion collections generated by this method span almost all of the genome are available to fly researchers. Alternatively, RNA interference can be used to effectively down-regulate fly genes in vivo using binary expression systems (outlined in the next section). Interestingly, genome-wide RNAi screens blur the distinction between forward and reverse genetics.

Lastly, homologous recombination can be used to specifically target genes to be knocked-out or replaced ("knocked-in") with other genes or alleles of interest (Rong et al., 2002). These genetic modifications are initially time-consuming and laborious due to the low efficiency of homologous recombination in flies. However, once the gene of interest is replaced with a genetic construct that allows for cassette exchange, subsequent knock-in experiments are far more efficient (Choi et al., 2009).

Finally, modifier screens combine forward and reverse genetics to identify interactions between genes (St Johnston, 2002). In this approach, a reverse-engineered mutant with a specific phenotype serves as "sensitized background", which is then crossed with randomly mutagenized flies or RNAi lines to identify genes that suppress or enhance the phenotype of interest. Because this type of screen reveals epistatic interactions, it can identify genes that normally would not show up in a traditional forward-genetics screen. For example, this approach has been useful for the discovery of several genetic modifiers for MeCP2 (Cukier et al., 2008), the gene responsible for most cases of Rett's Syndrome. This study nicely illustrates a broadly used approach for identifying pathways for human genes that do not have homologs in Drosophila. The authors overexpressed MeCP2 in the fly eye, which causes defects in eye development. They subsequently used a candidate gene approach to search for genes whose dosage reduction compensates for $\mathrm{MeCP} 2$ overexpression, identifying several interactors in the process. Follow up studies with the interacting gene osa suggest that $M e C P 2$ gain of function affects dendrite morphology in neurons (Vonhoff et al., 2012).

The screening approaches outlined above have led to the isolation of many key genes involved in the biology of the nervous system, such as neuronal specification, growth cone guidance, synaptic transmission, circadian rhythmicity, learning and memory (Dudai et al., 1976; Doe, 2008; Hartenstein et al., 2008; Vactor et al., 1993; Seeger et al., 1993; Suzuki et al., 1971; Konopka and Benzer, 1971; Cirelli, 2003). Of particular relevance to NDD studies, learning and memory mutants identified in the fly have been proposed to reveal putative ID genes (Morley and Montgomery, 2001). In fact, several genes such as cheerio and rsk identified in unbiased screens for learning and memory (Putz et al., 2004; Dubnau et al., 2003) in flies have human homologs implicated in ID disorders, clearly demonstrating the power of fly screening approaches in the identification of putative NDD genes.

\section{Targeted control of gene expression to unravel gene function}

As mentioned earlier, the nervous system has evolved to be as robust as possible to environmental and genetic changes. This means that the nervous system - and the organism as a whole works as hard as possible to adapt to the inevitable unpredictability of its genome and the environment. However, from a technical point of view, this robustness can mask phenotypes that reveal the true role that a gene plays in development. Therefore, a large fly genetic toolbox has been created for reverse genetic approaches to allow cell-type and developmental-stage specific control of gene expression (Venken et al., 2011). By downregulating or increasing a specific gene's expression in specific cell-types, we can study phenotypes that the system's inherent mechanisms of robustness would otherwise obscure. Moreover, spatio-temporal genetic control allows analysis of essential genes, which if mutated throughout the entire organism would cause lethality. Below we briefly outline some technical aspects of these tools.

The most commonly used elements in the fly genetic toolbox are microbially-derived binary expression systems. These expression systems consist of two genetic elements: an exogenous transcription factor and a synthetic promoter containing its binding sequence. A specific endogenous fly gene promoter drives expression of the transcription factor. In turn, this transcription factor specifically binds to a synthetic promoter to drive the expression of the selected transgene. This can be simply a gene of interest, a marker like a fluorescent protein, or an RNAi construct to target 
a particular endogenous gene for down-regulation. The main binary system used in the fly involves the yeast-derived transcription factor Gal4, which binds to the Upstream Activator Sequence (UAS) to drive transgene expression (Brand and Perrimon, 1993). Other recently developed binary expression systems such as the LexAop and QF:QUAS system can be used in parallel with the Gal4-UAS system to achieve more complex expression dynamics (Szuts and Bienz, 2000; Lai and Lee, 2006; Potter et al., 2010). The advent of new binary expression systems that do not cross-react means that different transgenes, such as markers, RNAi constructs and overexpression constructs can be expressed simultaneously in exclusive or overlapping patterns. This opens the door to examining genetic interactions and cellular interactions in parallel. The Q system is particularly promising because, like the Gal4 system and its repressor Gal80, includes a repressor protein, QS. Furthermore, it can be de-repressed using a chemical, Quinic Acid (QA) which adds another layer of control.

Endogenous promoters differentially drive expression of genes in specific cell types, during specific life stages. Binary expression systems take advantage of this to provide spatio-temporal control of the gene studied, which greatly expands the potential for analysis of gene function. The use of binary expression systems has been critical in the study of NDD genes in Drosophila; for example, the UAS-Gal4 system has been used extensively to characterize neuronal morphology defects in the fmr mutant fly (Fig. 1).

By restricting the expression of a transgene only to cells of interest, at a select developmental stage, one can also overcome a number of technical obstacles, such as the ability to visualize and modulate activity in distinct cellular populations. For instance, by coupling the Gal4 transcription factor to a gene promoter specifically expressed in a neuronal or glial population, one can drive the expression of a reporter gene such as GFP, to selectively visualize these cells. Depending on the localization properties of the reporter gene, the system can be used to mark specific sub-cellular structures. This is achieved by fusing the UAS-coupled fluorescent reporter gene with a compartmental protein which localizes to the cell membrane, cytoplasm, mitochondria, nucleus, nucleolus, dendrites, synapse, ER or Golgi. Labeling groups of cells and specific cellular structures provides the ability to assess loss of function phenotypes within specific populations of neuronal cells. For instance this approach is used in the fly model of Fragile $\mathrm{X}$ syndrome to reveal excessive dendritic and axonal over-elaboration in a number of neuronal populations (Fig. 1).

Numerous Gal4 lines with characterized expression patterns have become available to fly researchers through commercial fly stocks. In addition, research groups in Janelia Farm (HHMI Janelia Farm, VA, US) have recently generated thousands of novel Gal4 lines, and are in the process of characterizing the spatio-temporal pattern of expression associated with each stock (Pfeiffer et al., 2008). These efforts are likely to produce enough lines with distinct expression patterns to allow analysis of the majority of neuronal and glial populations in the brain.

Using Gal4 elements to drive expression of reporters is also useful for discovering populations of cells in which a certain gene is expressed and establishing their function in driving behavior. To identify which cells express a gene of interest, a Gal4 line with the promoter of a gene with known function can simply drive expression of a GFP reporter, illuminating all cells which express that gene. This approach has been used to characterize and group classes of pacemaker neurons involved in circadian rhythm of flies (Shafer et al., 2008).

Using similar approaches, binary expression systems can be used to alter cell physiology within specific neuronal populations (Venken et al., 2011; Keene and Waddell, 2007). For instance, Gal4 lines can be used to drive the expression of exogenous toxins or proapaptotic genes to eliminate a select set of neurons, and thereafter observe the functional and behavioral consequences. Alternatively, altering the expression of specific ion channels in a neuronal population can enhance or hamper electrophysical activity in these cells. These powerful techniques that modulate the activity of certain population of cells helps understand their niche within the nervous system, how they participate in development and how they affect behavior.

Binary expression systems in the fly can be further regulated by extrinsic factors acting on the system, such as temperature, thereby providing a temporal control of transgene expression. For example,

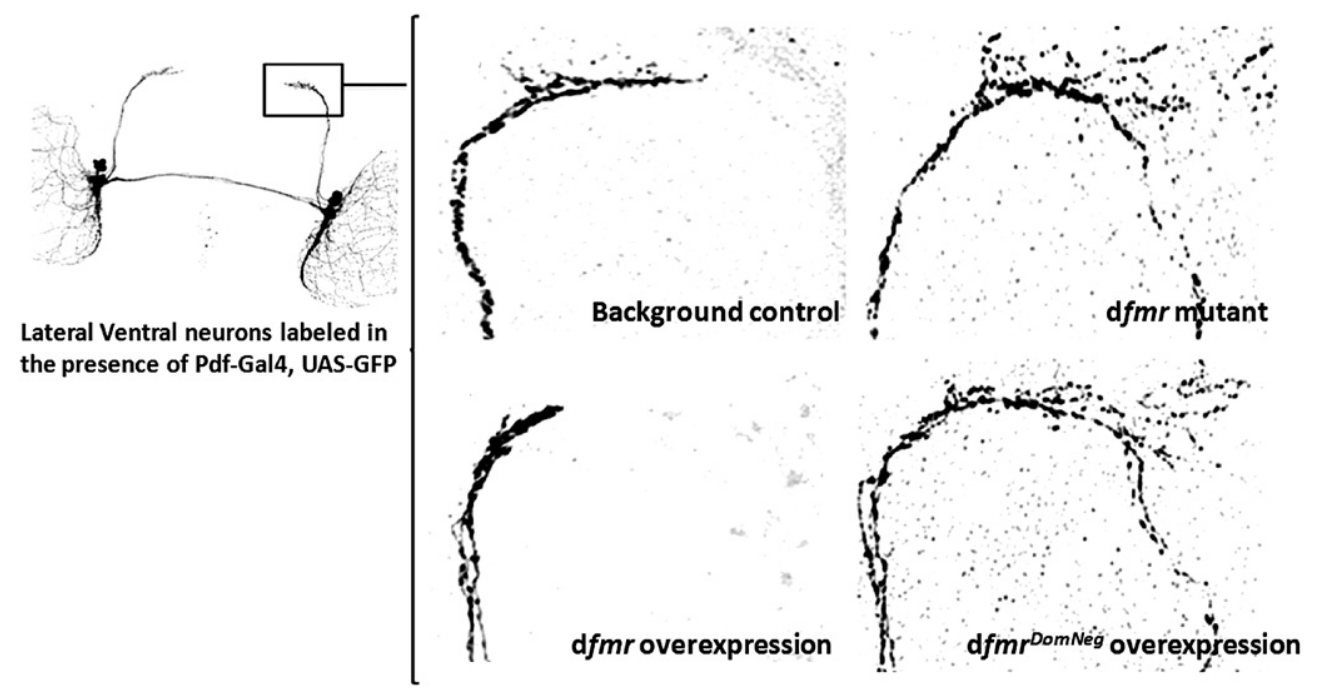

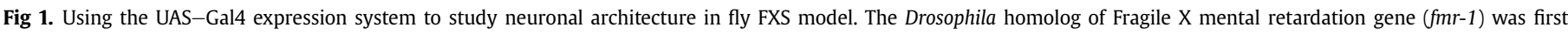

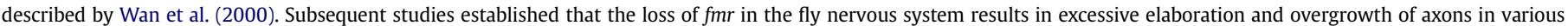

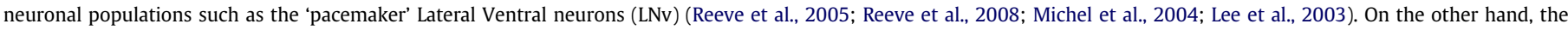

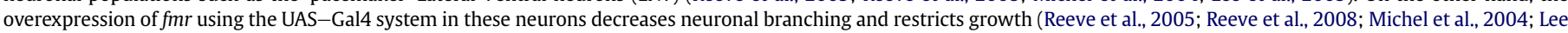

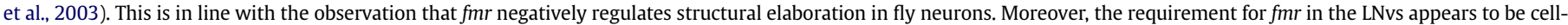

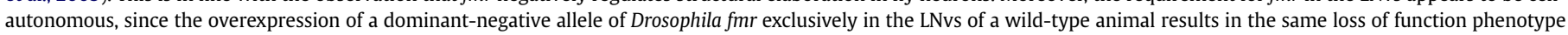
observed in the full mutant (Reeve et al., 2008). 
a temperature sensitive allele exists for Gal80 - a repressor of the Gal4 transcriptional activator - and so can be used to inhibit Gal4 expression only at permissive temperatures (McGuire et al., 2003). Another way to interfere with driver expression is to use druginducible Gal4 systems (Stebbins et al., 2001; Roman et al., 2001). Having temporal control over gene expression helps uncouple developmental defects from adult-specific defects in a mutant, which is a crucial step in understanding NDD etiology and devising potential therapeutics. For instance, the drug inducible GeneSwitch system has revealed that fly memory defects observed in the absence of the fly homolog of Neurofibramatosis type 1 (dNF1) is mostly an adult stage defect, and can be rescued with the expression of $d N F 1$ specifically in the adult stage (Buchanan and Davis, 2010).

Finally, binary expression systems can be used to induce genome-level disruptions in single cells within the brain (Venken et al., 2011). In particular, controlled expression of the yeast FLP recombinase can cause precise mitotic recombination between sister chromatids at specifically engineered FRT sites. If done in a background that is heterozygous for the recombined gene region, one of the resulting daughter cells - that is now homozygous for the mutation - can be directly examined within its organic or developmental setting (Lee and Luo, 2001). This method is particularly useful for studying the stage-specific, cell-autonomous requirement of a gene with diverse functions in different tissues and at different developmental stages. For instance, clonal analyses using this system have revealed the role of Drosophila fmr in the proliferation capacity of neural stem cells in the brain: fmr null neuroblasts give rise to more neurons compared to the control neuroblasts in the same organism (Callan et al., 2010).

The FRT-FLP system is also useful for the stochastic labeling of cells. As mentioned above, a Gal4 element can be used to drive the expression of a reporter gene in a specific population of neurons. Introducing FLP-FRT into this system can help restrict the expression of the reporter gene to only a single or few cells. For instance, engineering a stop cassette - flanked by FRT sites between the UAS element and the reporter gene can block the expression of the reporter in all Gal4 expressing cells, except in those with induced FLP gene expression. The stochastic labeling of a few cells helps visualize neuronal architecture with single-cell resolution as well as allowing for lineage tracing of cells (Hadjieconomou et al., 2011; Lee, 2009).

\section{Conclusion}

D. melanogaster is established as a valuable research organism for the study of many human diseases including neurodevelopmental disorders (Bellen et al., 2010; Bier, 2005; Leyssen and Hassan, 2007). The power of the fly model in investigating NDD etiology stems from the fact that the genetic and molecular logic, and basic architecture of its nervous system is, to a very large extent, shared by mammals. In addition, the experimental tractability of the fly surpasses that of all higher eukaryote models; an extensive collection of genetic tools and experimental techniques allows us to thoroughly investigate gene function at the cellular and behavioral level. Flies are easy to rear in large numbers, which gives the possibility to do large-scale experiments and use screening approaches. Genetic screens are extremely effective in identifying novel genes, their function in the nervous system and their particular context within a molecular pathway. Thus, the fruit fly model is optimal for initial discovery and characterization of NDD genes and molecular pathways that can then be further investigated in the mammalian context. Finally, an emerging trend in fly research is to investigate gene-environment interactions and its influence on the phenotypic outcome. This is likely to turn out to be an important aspect of studying NDDs, due to its implications regarding pre-natal care and educational opportunities for individuals suffering from these disorders.

\section{Acknowledgments}

We would like to thank Aaron New for his comments on the manuscript. We would also like to thank Dr. Patrick Callaerts and Dr. Patrik Verstreken for helpful discussions.

\section{References}

Acampora, D., et al., 1996. Epilepsy and brain abnormalities in mice lacking the Otx1 gene. Nat. Genet. 14 (2), 218-222.

Acampora, D., et al., 1998. Murine Otx1 and Drosophila otd genes share conserved genetic functions required in invertebrate and vertebrate brain development. Development 125 (9), 1691-1702.

Akten, B., et al., 2009. Ribosomal s6 kinase cooperates with casein kinase 2 to modulate the Drosophila circadian molecular oscillator. J. Neurosci. 29 (2), 466-475.

Al-Tawashi, A., et al. A protein implicated in non-syndromic mental retardation regulates the protein kinase a (PKA) activity. J. Biol. Chem.

Araujo, S.J., Tear, G., 2003. Axon guidance mechanisms and molecules: lessons from invertebrates. Nat. Rev. Neurosci. 4 (11), 910-922.

Banerjee, P., et al., 2010. Short- and long-term memory are modulated by multiple isoforms of the fragile X mental retardation protein. J. Neurosci. 30 (19), 6782-6792.

Bellen, H.J., Tong, C., Tsuda, H., 2010. 100 years of Drosophila research and its impact on vertebrate neuroscience: a history lesson for the future. Nat. Rev. Neurosci. 11 (7), 514-522.

Bier, E., 2005. Drosophila, the golden bug, emerges as a tool for human genetics. Nat. Rev. Genet. 6 (1), 9-23.

Bolduc, F.V., et al., 2008. Excess protein synthesis in Drosophila fragile X mutants impairs long-term memory. Nat. Neurosci. 11 (10), 1143-1145.

Bolduc, F.V., Tully, T., 2009. Fruit flies and intellectual disability. Fly (Austin) 3 (1), 91-104

Bonini, N.M., 2000. Drosophila as a genetic tool to define vertebrate pathway players. Methods Mol. Biol. 136, 7-14.

Bourtchouladze, R., et al., 2003. A mouse model of Rubinstein-Taybi syndrome: defective long-term memory is ameliorated by inhibitors of phosphodiesterase 4. Proc. Natl. Acad. Sci. U. S. A. 100 (18), 10518-10522.

Brand, A.H., Perrimon, N., 1993. Targeted gene expression as a means of altering cell fates and generating dominant phenotypes. Development 118 (2), 401-415.

Buchanan, M.E., Davis, R.L., 2010. A distinct set of Drosophila brain neurons required for neurofibromatosis type 1-dependent learning and memory. J. Neurosci. 30 (30), 10135-10143.

Callan, M.A., et al., 2010. Fragile X protein controls neural stem cell proliferation in the Drosophila brain. Hum. Mol. Genet. 19 (15), 3068-3079.

Chien, S., et al., 2002. Homophila: human disease gene cognates in Drosophila. Nucleic Acids Res. 30 (1), 149-151.

Choi, C.M., et al., 2009. Conditional mutagenesis in Drosophila. Science 324 (5923), 54.

Choi, C.H., et al., 2010. Age-dependent cognitive impairment in a Drosophila fragile $X$ model and its pharmacological rescue. Biogerontology 11 (3), 347-362.

Cirelli, C., 2003. Searching for sleep mutants of Drosophila melanogaster. Bioessays 25 (10), 940-949.

Cirelli, C., Bushey, D., 2008. Sleep and wakefulness in Drosophila melanogaster. Ann. N. Y. Acad. Sci. 1129, 323-329.

Coffee Jr., R.L., et al., 2011. In vivo neuronal function of the fragile X mental retardation protein is regulated by phosphorylation. Hum. Mol. Genet. 21 (4), 900-915.

Costa, A., et al., 2005. The Drosophila fragile X protein functions as a negative regulator in the orb autoregulatory pathway. Dev. Cell 8 (3), 331-342.

Cukier, H.N., et al., 2008. Genetic modifiers of MeCP2 function in Drosophila. PLoS Genet. 4 (9), e1000179.

Dankert, H., et al., 2009. Automated monitoring and analysis of social behavior in Drosophila. Nat. Methods 6 (4), 297-303.

Dickson, B.J., 2002. Molecular mechanisms of axon guidance. Science 298 (5600), 1959-1964.

Didelot, G., et al., 2006. Tequila, a neurotrypsin ortholog, regulates long-term memory formation in Drosophila. Science 313 (5788), 851-853.

Dockendorff, T.C., et al., 2002. Drosophila lacking dfmr1 activity show defects in circadian output and fail to maintain courtship interest. Neuron 34 (6), 973-984

Doe, C.Q., 2008. Neural stem cells: balancing self-renewal with differentiation. Development 135 (9), 1575-1587.

Dubnau, J., et al., 2003. The staufen/pumilio pathway is involved in Drosophila longterm memory. Curr. Biol. 13 (4), 286-296.

Dudai, Y., et al., 1976. dunce, a mutant of Drosophila deficient in learning. Proc. Natl. Acad. Sci. U. S. A. 73 (5), 1684-1688.

Epstein, A.M., et al., 2009. Drosophila Fragile X protein controls cellular proliferation by regulating cbl levels in the ovary. Dev. Biol. 330 (1), 83-92.

Ferdousy, F., et al., 2011. Drosophila Ube3a regulates monoamine synthesis by increasing GTP cyclohydrolase I activity via a non-ubiquitin ligase mechanism. Neurobiol. Dis. 41 (3), 669-677.

Fischer, M., et al., 2009. Drosophila RSK negatively regulates bouton number at the neuromuscular junction. Dev. Neurobiol. 69 (4), 212-220. 
Gatto, C.L., Broadie, K., 2011. Drosophila modeling of heritable neurodevelopmental disorders. Curr. Opin. Neurobiol. 21 (6), 834-841.

Ghysen, A., 2003. The origin and evolution of the nervous system. Int. J. Dev. Biol. 47 (7-8), 555-562.

Greenspan, R.J., Dierick, H.A., 2004. 'Am not I a fly like thee?' From genes in fruit flies to behavior in humans. Hum. Mol. Genet. 13 Spec No 2, R267-R273.

Greenspan, R.J., van Swinderen, B., 2004. Cognitive consonance: complex brain functions in the fruit fly and its relatives. Trends Neurosci. 27 (12), 707-711.

Guo, H.F., et al., 1997. Requirement of Drosophila NF1 for activation of adenylyl cyclase by PACAP38-like neuropeptides. Science 276 (5313), 795-798.

Guo, H.F., et al., 2000. A neurofibromatosis-1-regulated pathway is required for learning in Drosophila. Nature 403 (6772), 895-898.

Hadjieconomou, D., et al., 2011. Flybow: genetic multicolor cell labeling for neural circuit analysis in Drosophila melanogaster. Nat. Methods 8 (3), 260-266.

Hardin, P.E., 2005. The circadian timekeeping system of Drosophila. Curr. Biol. 15 (17), R714-R722.

Hartenstein, V., et al., 2008. The development of the Drosophila larval brain. Adv Exp. Med. Biol. 628, 1-31.

Heberlein, U., 2000. Genetics of alcohol-induced behaviors in Drosophila. Alcohol. Res. Health 24 (3), 185-188.

Heberlein, U., et al., 2009. Drosophila, a genetic model system to study cocainerelated behaviors: a review with focus on LIM-only proteins. Neuropharmacology 56 (Suppl. 1), 97-106.

Hirth, F., et al., 2003. An urbilaterian origin of the tripartite brain: developmental genetic insights from Drosophila. Development 130 (11), 2365-2373.

Inlow, J.K., Restifo, L.L., 2004. Molecular and comparative genetics of mental retardation. Genetics 166 (2), 835-881.

Inoue, S., et al., 2002. A role for the Drosophila fragile X-related gene in circadian output. Curr. Biol. 12 (15), 1331-1335.

Keene, A.C., Waddell, S., 2007. Drosophila olfactory memory: single genes to complex neural circuits. Nat. Rev. Neurosci. 8 (5), 341-354.

Kelley, D.J., et al., 2007. The cyclic AMP cascade is altered in the fragile $\mathrm{X}$ nervous system. PLoS One 2 (9), e931.

Kim, M., et al., 2006. Inhibition of ERK-MAP kinase signaling by RSK during Drosophila development. EMBO J. 25 (13), 3056-3067.

Konopka, R.J., Benzer, S., 1971. Clock mutants of Drosophila melanogaster. Proc. Natl. Acad. Sci. U. S. A. 68 (9), 2112-2116.

Kramer, J.M., et al. Epigenetic regulation of learning and memory by Drosophila EHMT/G9a. PLoS Biol. 9 (1), e1000569.

Lai, S.L., Lee, T., 2006. Genetic mosaic with dual binary transcriptional systems in Drosophila. Nat. Neurosci. 9 (5), 703-709.

Lee, T., 2009. New genetic tools for cell lineage analysis in Drosophila. Nat. Methods $6(8), 566-568$.

Lee, A., et al., 2003. Control of dendritic development by the Drosophila fragile Xrelated gene involves the small GTPase Rac1. Development 130 (22), 5543-5552.

Lee, T., Luo, L., 2001. Mosaic analysis with a repressible cell marker (MARCM) for Drosophila neural development. Trends Neurosci. 24 (5), 251-254.

Leyssen, M., Hassan, B.A., 2007. A fruitfly's guide to keeping the brain wired. EMBO Rep. 8 (1), 46-50.

Littleton, J.T., 2000. A genomic analysis of membrane trafficking and neurotransmitter release in Drosophila. J. Cell. Biol. 150 (2), F77-F82.

Littleton, J.T., Ganetzky, B., 2000. Ion channels and synaptic organization: analysis of the Drosophila genome. Neuron 26 (1), 35-43.

Lu, Y., et al., 2009. The Drosophila homologue of the Angelman syndrome ubiquitin ligase regulates the formation of terminal dendritic branches. Hum. Mol. Genet. 18 (3), 454-462.

Manev, H., Dimitrijevic, N., Dzitoyeva, S., 2003. Techniques: fruit flies as models for neuropharmacological research. Trends Pharmacol. Sci. 24 (1), 41-43.

McBride, S.M., et al., 2005. Pharmacological rescue of synaptic plasticity, courtship behavior, and mushroom body defects in a Drosophila model of fragile $X$ syndrome. Neuron 45 (5), 753-764.

McGuire, S.E., et al., 2003. Spatiotemporal rescue of memory dysfunction in Drosophila. Science 302 (5651), 1765-1768.

Michel, C.I., Kraft, R., Restifo, L.L., 2004. Defective neuronal development in the mushroom bodies of Drosophila fragile X mental retardation 1 mutants. J. Neurosci. 24 (25), 5798-5809.

Mironov, S.L., Skorova, E.Y., Kugler, S. Epac-mediated cAMP-signalling in the mouse model of Rett Syndrome. Neuropharmacology 60 (6), 869-877.

Morales, J., et al., 2002. Drosophila fragile X protein, DFXR, regulates neuronal morphology and function in the brain. Neuron 34 (6), 961-972.

Morley, K.I., Montgomery, G.W., 2001. The genetics of cognitive processes: candidate genes in humans and animals. Behav. Genet. 31 (6), 511-531.

Nagao, T., et al., 1998. Developmental rescue of Drosophila cephalic defects by the human Otx genes. Proc. Natl. Acad. Sci. U. S. A. 95 (7), 3737-3742.

Nichols, C.D., 2006. Drosophila melanogaster neurobiology, neuropharmacology, and how the fly can inform central nervous system drug discovery. Pharmacol. Ther. 112 (3), 677-700.

Ohno, S., 1970. Evolution of Gene Duplication. Springer.

Pan, L., et al., 2004. The Drosophila fragile X gene negatively regulates neuronal elaboration and synaptic differentiation. Curr. Biol. 14 (20), 1863-1870.

Papoulas, O., et al., 2010. dFMRP and Caprin, translational regulators of synaptic plasticity, control the cell cycle at the Drosophila mid-blastula transition. Development 137 (24), 4201-4209.

Pfeiffer, B.D., et al., 2008. Tools for neuroanatomy and neurogenetics in Drosophila. Proc. Natl. Acad. Sci. U. S. A. 105 (28), 9715-9720.
Pitman, J.L., et al., 2009. There are many ways to train a fly. Fly (Austin) 3 (1), 3-9. Potter, C.J., et al., 2010. The $\mathrm{Q}$ system: a repressible binary system for transgene expression, lineage tracing, and mosaic analysis. Cell 141 (3), 536-548.

Putz, G., et al., 2004. The S6KII (rsk) gene of Drosophila melanogaster differentially affects an operant and a classical learning task. J. Neurosci. 24 (44), 9745-9751.

Reeve, S.P., et al., 2005. The Drosophila fragile X mental retardation protein controls actin dynamics by directly regulating profilin in the brain. Curr. Biol. 15 (12), 1156-1163.

Reeve, S.P., et al., 2008. Mutational analysis establishes a critical role for the N terminus of fragile X mental retardation protein FMRP. J. Neurosci. 28 (12), 3221-3226.

Reichert, H., 2002. Conserved genetic mechanisms for embryonic brain patterning. Int. J. Dev. Biol. 46 (1), 81-87.

Reichert, H., Simeone, A., 1999. Conserved usage of gap and homeotic genes in patterning the CNS. Curr. Opin. Neurobiol. 9 (5), 589-595.

Reiter, L.T., et al., 2001. A systematic analysis of human disease-associated gene sequences in Drosophila melanogaster. Genome Res. 11 (6), 1114-1125.

Restifo, L.L., 2005. Mental retardation genes in drosophila: new approaches to understanding and treating developmental brain disorders. Ment. Retard. Dev. Disabil. Res. Rev. 11 (4), 286-294.

Robinson, D.N., et al., 1997. Formation of the Drosophila ovarian ring canal inner rim depends on cheerio. Genetics 145 (4), 1063-1072.

Roman, G., et al., 2001. P[Switch], a system for spatial and temporal control of gene expression in Drosophila melanogaster. Proc. Natl. Acad. Sci. U S A 98 (22) $12602-12607$.

Romeo, Y., Zhang, X., Roux, P.P., 2012. Regulation and function of the RSK family of protein kinases. Biochem. J. 441 (2), 553-569.

Rong, Y.S., et al., 2002. Targeted mutagenesis by homologous recombination in D. melanogaster. Genes Dev. 16 (12), 1568-1581.

Rubin, G.M., et al., 2000. Comparative genomics of the eukaryotes. Science 287 (5461), 2204-2215.

Rus, F., et al., 2006. Expression pattern of Filamin-240 in Drosophila blood cells. Gene Expr. Patterns 6 (8), 928-934.

Sanchez-Soriano, N., et al., 2005. Are dendrites in Drosophila homologous to vertebrate dendrites? Dev. Biol. 288 (1), 126-138.

Sancini, G., et al., 2001. Potentially epileptogenic dysfunction of cortical NMDA- and GABA-mediated neurotransmission in Otx1-/- mice. Eur. J. Neurosci. 14 (7) 1065-1074.

Schenck, A., et al., 2003. CYFIP/Sra-1 controls neuronal connectivity in Drosophila and links the Rac1 GTPase pathway to the fragile X protein. Neuron 38 (6), 887-898.

Seeger, M., et al., 1993. Mutations affecting growth cone guidance in Drosophila: genes necessary for guidance toward or away from the midline. Neuron 10 (3), 409-426.

Shafer, O.T., et al., 2008. Widespread receptivity to neuropeptide PDF throughout the neuronal circadian clock network of Drosophila revealed by real-time cyclic AMP imaging. Neuron 58 (2), 223-237.

Sokol, N.S., Cooley, L., 1999. Drosophila filamin encoded by the cheerio locus is a component of ovarian ring canals. Curr. Biol. 9 (21), 1221-1230.

Sokol, N.S., Cooley, L., 2003. Drosophila filamin is required for follicle cell motility during oogenesis. Dev. Biol. 260 (1), 260-272.

St Johnston, D., 2002. The art and design of genetic screens: drosophila melanogaster. Nat. Rev. Genet. 3 (3), 176-188.

Stebbins, M.J., et al., 2001. Tetracycline-inducible systems for Drosophila. Proc. Natl. Acad. Sci. U. S. A. 98 (19), 10775-10780.

Steffan, J.S., et al., 2001. Histone deacetylase inhibitors arrest polyglutaminedependent neurodegeneration in Drosophila. Nature 413 (6857), 739-743.

Suzuki, D.T., Grigliatti, T., Williamson, R., 1971. Temperature-sensitive mutations in Drosophila melanogaster. VII. A mutation (para-ts) causing reversible adult paralysis. Proc. Natl. Acad. Sci. U. S. A. 68 (5), 890-893.

Szuts, D., Bienz, M., 2000. LexA chimeras reveal the function of Drosophila Fos as a context-dependent transcriptional activator. Proc. Natl. Acad. Sci. U. S. A. 97 (10), 5351-5356

The, I., et al., 1997. Rescue of a Drosophila NF1 mutant phenotype by protein kinase A. Science 276 (5313), 791-794.

Thor, S., 1995. The genetics of brain development: conserved programs in flies and mice. Neuron 15 (5), 975-977.

Vactor, D.V., et al., 1993. Genes that control neuromuscular specificity in Drosophila. Cell 73 (6), 1137-1153.

Venken, K.J., Simpson, J.H., Bellen, H.J., 2011. Genetic manipulation of genes and cells in the nervous system of the fruit fly. Neuron 72 (2), 202-230.

Vonhoff, F., et al., 2012. Drosophila as a model for MECP2 gain of function in neurons. PLoS One 7 (2), e31835.

Vosshall, L.B., 2007. Into the mind of a fly. Nature 450 (7167), 193-197.

Wan, L., et al., 2000. Characterization of dFMR1, a Drosophila melanogaster homolog of the fragile X mental retardation protein. Mol. Cell. Biol. 20 (22), 8536-8547.

Wang, V.Y., et al., 2002. Drosophila atonal fully rescues the phenotype of Math1 nul mice: new functions evolve in new cellular contexts. Curr. Biol. 12 (18), 1611-1616.

Williams, J.A., et al., 2001. A circadian output in Drosophila mediated by neurofibromatosis-1 and Ras/MAPK. Science 293 (5538), 2251-2256.

Wu, Y., et al., 2008. A Drosophila model for Angelman syndrome. Proc. Natl. Acad. Sci. U. S. A. 105 (34), 12399-12404.

$\mathrm{Xu}, \mathrm{X}$.L., et al., 2008. The steady-state level of the nervous-system-specific microRNA124a is regulated by dFMR1 in Drosophila. J. Neurosci. 28 (46), 11883-11889.

Zhang, S.X., Guo, Y., Boulianne, G.L., 2001. Identification of a novel family of putative methyltransferases that interact with human and Drosophila presenilins. Gene $280(1-2), 135-144$

Zheng, L., et al., 2011. Drosophila Ten-m and filamin affect motor neuron growth cone guidance. PLoS One 6 (8), e22956. 